\title{
Selective Correlation of Amide Groups to Glycine Alpha Protons and of Arginine Guanidine Groups to Delta Protons in Proteins by Multiple Quantum Spectroscopy
}

\author{
Renzo Bazzo, ${ }^{1}$ Daniel O. Cicero, and Gaetano Barbato \\ Istituto di Ricerca di Biologia Molecolare P. Angeletti spa, Via Pontina km 30.600, Pomezia, 00040, Rome, Italy
}

Received February 27, 1998; revised July 23, 1998

\begin{abstract}
A new 3D pulse sequence correlates backbone amide proton and nitrogen with alpha proton resonances selectively for glycine residues in a fully doubly labeled $\left({ }^{15} \mathrm{~N},{ }^{13} \mathrm{C}\right)$ protein. The excitation of multiple quantum coherences provides optimized resolution and sensitivity. D egenerate alpha proton groups can be promptly recognized. Correlation of guanidine $\mathrm{N} \mathrm{H}$ groups to delta protons of arginine side chains is also obtained. ๑ 1999 Academic Press
\end{abstract}

Key Words: NMR; Gly; protein; multiple quantum; strong coupling.

\section{INTRODUCTION}

Several triple-resonance three- and four-dimensional techniques have been developed in recent years to achieve throughbond connectivities among ${ }^{1} \mathrm{H}$ and ${ }^{15} \mathrm{~N}$ resonances of amide groups and the intraresidue and sequential ${ }^{13} \mathrm{C}_{\alpha}$ and ${ }^{1} \mathrm{H}_{\alpha}$ resonances (1-3).

The more convenient approach takes advantage of the favorable relaxation properties of the amide ${ }^{15} \mathrm{~N}$ compared to the ${ }^{13} \mathrm{C}_{\alpha}$ and transfers amide proton magnetization through ${ }^{15} \mathrm{~N}$ and ${ }^{13} \mathrm{C}_{\alpha}$ to the ${ }^{1} \mathrm{H}_{\alpha}$ and then back to ${ }^{1} \mathrm{H}_{\mathrm{N}}(2,3)$. In any case, these methods are designed (or optimized) to perform such correlation for nonglycine residues, in that one always assumes that a single proton is directly coupled to the ${ }^{13} \mathrm{C}_{\alpha}$. As a result, the backbone connectivity is often interrupted at glycine residues, preventing complete sequential assignment (4). To circumvent this difficulty, a 3D selective correlation experiment was recently proposed which works uniquely on glycines, among all other residues (5). The magnetization transfer pathway follows the scheme outlined above in which the magnetization is transferred to the ${ }^{1} \mathrm{H}_{\alpha}$ atoms from the $\mathrm{C}_{\alpha}$ atoms via the ${ }^{1} J_{\mathrm{C}_{\alpha} \mathrm{H}_{\alpha}}$ coupling. For our purposes it is sufficient to restrict the analysis to the generation and evolution $\left(t_{1}\right)$ of the ${ }^{1} \mathrm{H}_{\alpha}$ resonances. In the product-operator formalism (6) we will use the following operator symbols: $\mathrm{N}$ for ${ }^{15} \mathrm{~N}$ spin, $\mathrm{C}$ for ${ }^{13} \mathrm{C}_{\alpha}$ spin, and I for

\footnotetext{
${ }^{1}$ To whom correspondence should be addressed.
}

${ }^{1} \mathrm{H}_{\alpha}$ spin. The two ${ }^{1} \mathrm{H}_{\alpha}$ atoms of glycines are indicated by $\mathrm{I}_{1}$ and $\mathrm{I}_{2}$. For glycines the state before the coherence transfer at the onset of $t_{1}$ evolution can be represented, omitting constant numerical factors, by

$$
\rho(a)=\mathrm{N}_{z} \mathrm{C}_{y} \mathrm{I}_{1 z} \mathrm{I}_{2 z},
$$

where $\rho(a)$ stands for the spin density matrix. This term is partially converted into transverse proton single quantum coherences by $90^{\circ}$ carbon and $45^{\circ}$ proton pulses (5), thereby generating

$$
\rho\left(t_{1}\right)=1 / 2 \mathrm{~N}_{z} \mathrm{C}_{z}\left\{\mathrm{I}_{1 y} \mathrm{I}_{2 z} \cos \left(2 \pi \nu_{1} t_{1}\right)+\mathrm{I}_{1 z} \mathrm{I}_{2 y} \cos \left(2 \pi \nu_{2} t_{1}\right)\right\} .
$$

Here and in the following we indicate only terms that are selected by the phase cycle. $\nu_{1}, \nu_{2}$ are the proton chemical shifts. At the end of the $t_{1}$ period, a second proton-carbon pulse pair similar to the previous one will produce

$$
\rho(b)=1 / 4 \mathrm{~N}_{z} \mathrm{C}_{y} \mathrm{I}_{1 z} \mathrm{I}_{2 z}\left\{\cos \left(2 \pi \nu_{1} t_{1}\right)+\cos \left(2 \pi \nu_{2} t_{1}\right)\right\},
$$

where $t_{1}$ needs to be short $\left(t_{1 \max }<10 \mathrm{~ms}\right)$ to neglect the effect of the geminal coupling.

For our purposes the term in Eq. [3] represents the amount of magnetization that will be finally converted back into observable signal according to this method (5).

In what follows we illustrate how, by following a different route, the observable signal can be enhanced more than twice in sensitivity, allowing also optimal resolution in the ${ }^{1} \mathrm{H}_{\alpha}$ dimension $\left(t_{1 \max }>10 \mathrm{~ms}\right)$. This new route brings about multiple quantum coherences in the $t_{1}$ period.

\section{THE NEW PULSE SCHEME}

The pulse sequence is illustrated in Fig. 1. We examine in detail the coherence transfer between points $\mathrm{a}$ and $\mathrm{b}$ which 

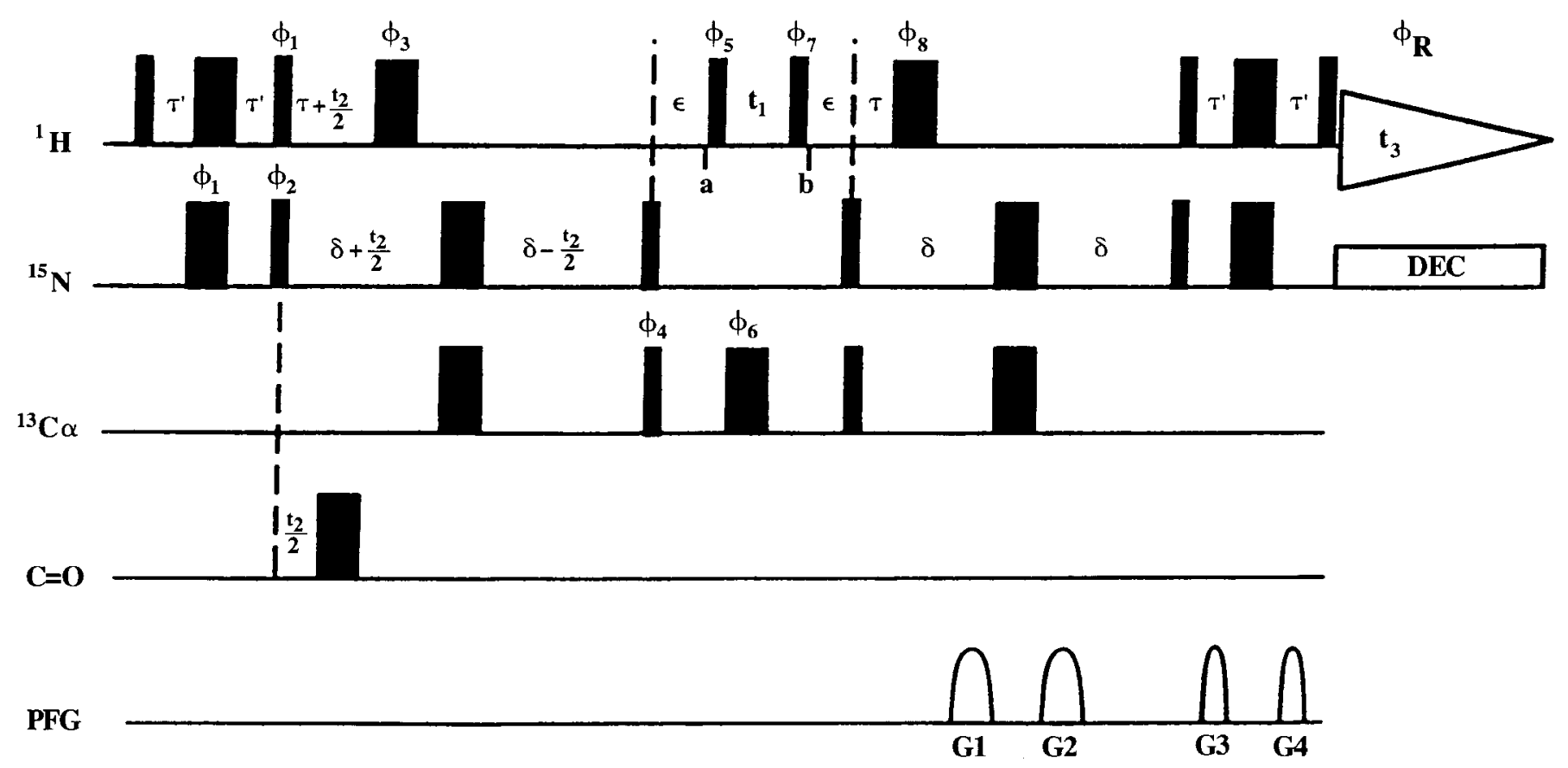

FIG. 1. 3D Pulse scheme used to correlate backbone amide protons to nitrogen and alpha protons, selectively for glycine residues in a doubly labeled protein. Wide and narrow solid rectangles indicate $180^{\circ}$ and $90^{\circ}$ pulses, respectively, with phase $x$ where not indicated. Phase cycling is as follows: $\phi_{1}=y,-y ; \phi_{2}=x, x,-x,-x ; \phi_{3}=x ; \phi_{4}=4(x), 4(-x) ; \phi_{5}=8(x), 8(-x) ; \phi_{6}=32(x), 32(-x) ; \phi_{7}=16(y), 16(-y) ; \phi_{8}=8(-x), 8(x) ;$ $\phi_{\mathrm{R}}=x,-x,-x, x,-x, x, x,-x$. The durations of the fixed delays are $\tau^{\prime}=2.5 \mathrm{~ms} ; \tau=2.75 \mathrm{~ms} ; \delta=12 \mathrm{~ms} ; \epsilon=3 \mathrm{~ms}$. $\phi_{2}$ is incremented according to the States-TPPI method; $\phi_{5}$ is not incremented. Complex FT is executed in $t_{1}$ and real FT is executed in $t_{2}$. Proton carrier is at low field with respect to ${ }^{1} \mathrm{H}_{\alpha}$ signals (see text for alternative detection methods). Sine-bell-shaped pulsed field gradients (PFG) with a strength of $8 \mathrm{G} / \mathrm{cm}$ at their maximum are applied along the $z$-axis with the following durations: $G_{1}=G_{2}=4 \mathrm{~ms} ; G_{3}=G_{4}=1.5 \mathrm{~ms}$. No water presaturation is used. See text for implementation of water selective flipback pulses.

enclose the $t_{1}$ evolution period of the alpha protons. At point $a$ in the sequence the system can be represented by the same expression used in Eq. [1]. The $90^{\circ}$ proton pulse $\phi_{5}$ will generate the term

$$
\rho\left(t_{1}\right)=\mathrm{N}_{z} \mathrm{C}_{y} \mathrm{I}_{1 y} \mathrm{I}_{2 y}
$$

which represents a linear combination of heteronuclear multiple quantum coherences. This term can be selected with respect to other coherences, belonging to nonglycine spin systems, simply by alternating the phase $\phi_{5}$. As it turns out, the equivalent $(\mathrm{NH})-\mathrm{CH}_{2}$ spin system present in arginine side chains get selected, too, although the resulting signal is easily distinguishable by the different shift and multiplet structure, due to the additional couplings with the subsequent methilene group.

The operatorial term expressed by Eq. [4] will now evolve during $t_{1}$, subject to the action of the Hamiltonian of the system. The analysis can be largely simplified if one considers that the operators describing nitrogen and carbon do not undergo any net evolution in $t_{1}$ since the $180^{\circ}$ alphacarbon pulse removes the effect of both alpha-carbon chem- ical shift evolution and its couplings with nitrogen and carbonyl atoms. The couplings ${ }^{1} J_{\mathrm{C}_{\alpha} \mathrm{H}_{1}},{ }^{1} J_{\mathrm{C}_{\alpha} \mathrm{H}_{2}}$, on the other hand, are not effective on the multiple quantum term expressed in Eq. [4]. Therefore, we simply focus on the proton part, $\rho_{\mathrm{H}}\left(t_{1}\right)=\mathrm{I}_{1 y} \mathrm{I}_{2 y}\left(t_{1}\right)$,

$$
\begin{aligned}
\mathrm{I}_{1 y} \mathrm{I}_{2 y}\left(t_{1}\right)=1 / 4 & \left\{\left[\mathrm{I}_{1}^{+} \mathrm{I}_{2}^{-}\left(t_{1}\right)+\mathrm{I}_{1}^{-} \mathrm{I}_{2}^{+}\left(t_{1}\right)\right]\right. \\
& \left.-\left[\mathrm{I}_{1}^{+} \mathrm{I}_{2}^{+}\left(t_{1}\right)+\mathrm{I}_{1}^{-} \mathrm{I}_{2}^{-}\left(t_{1}\right)\right]\right\},
\end{aligned}
$$

where the shift operators (7) in the square brackets are used to represent zero-quantum coherence (ZQC) and double-quantum coherence (2QC), respectively.

If $\mathrm{H}_{1}$ and $\mathrm{H}_{2}$ are weakly coupled $\left(J_{12} /\left(\nu_{1}-\nu_{2}\right) \ll 1\right)$, the expressions above can be directly used to describe ZQC and $2 \mathrm{QC}$, and the $t_{1}$ evolution results in

$$
\begin{aligned}
\rho_{\mathrm{H}}\left(t_{1}\right)=1 / 2\left\{\left[\mathrm{I}_{1 y} \mathrm{I}_{2 y}+\mathrm{I}_{1 x} \mathrm{I}_{2 x}\right] \cos \left(2 \pi \nu_{\mathrm{ZQC}} t_{1}\right)+\left[\mathrm{I}_{1 y} \mathrm{I}_{2 y}-\mathrm{I}_{1 x} \mathrm{I}_{2 x}\right]\right. \\
\left.\times \cos \left(2 \pi \nu_{2 \mathrm{QC}} t_{1}\right)\right\}+ \text { other terms, }
\end{aligned}
$$

with $\nu_{\mathrm{ZQC}}=\nu_{1}-\nu_{2}$ and $\nu_{2 \mathrm{QC}}=\nu_{1}+\nu_{2}$.

At the end of $t_{1}$, a second phase-alternated proton $90^{\circ}$ pulse 


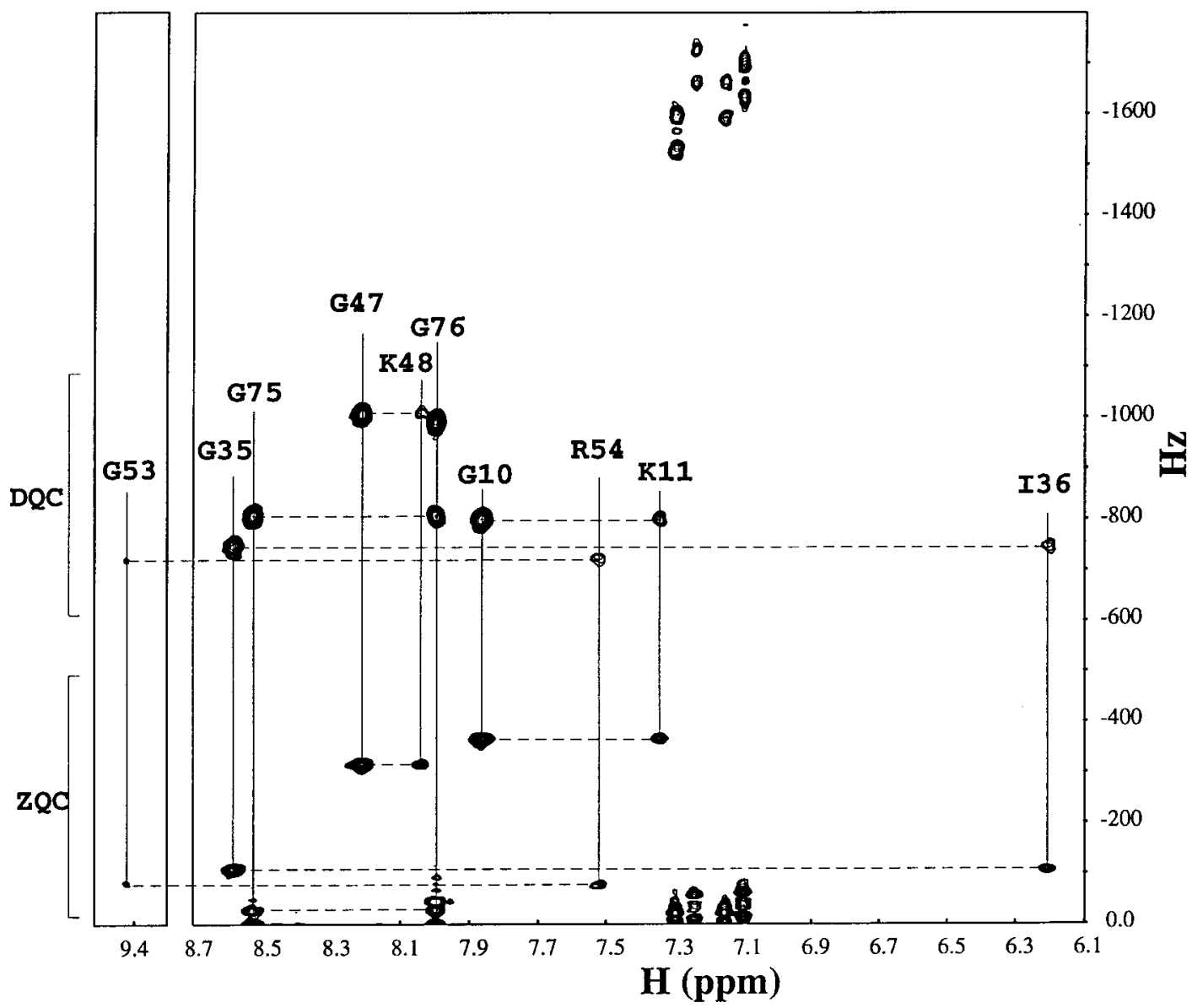

FIG. 2. $2 \mathrm{D}$ correlation spectrum between backbone amide proton and alpha proton resonances, selective for glycine residues of a doubly labeled $\left({ }^{15} \mathrm{~N}\right.$, $\left.{ }^{13} \mathrm{C}\right)$ sample of ubiquitin. The pulse sequence used is simply the $2 \mathrm{D}$ version of the $3 \mathrm{D}$ pulse scheme illustrated in Fig. 1, where the nitrogen evolution time is not incremented. The double-quantum peaks show up at a frequency corresponding to the sum of the proton shifts with respect to the carrier, positioned at 4.75 ppm. The zero-quantum peaks show up at a frequency that directly represents the chemical shift difference between the two alpha protons. A simple calculation provides the two alpha-proton chemical shifts that are correlated with the amide protons in the same residue $(i)$ and in the subsequent residue $(i+1)$, via the $\mathrm{C}_{i}-\mathrm{N}_{i} J_{1}$ and $\mathrm{C}_{i}-\mathrm{N}_{i+1} J_{2}$ coupling, respectively. The analogue peaks for arginine residues appear in a separate region of the spectrum and their frequency positions are to be taken in the center of the corresponding multiplet. Zero-frequency peaks show strong coupling effects for Gly 75 and Gly 76 (see text).

$\left(\phi_{7}\right)$ can select either of the two terms $\mathrm{I}_{1 y} \mathrm{I}_{2 y}$ or $\mathrm{I}_{1 x} \mathrm{I}_{2 x}$ to generate

$$
\rho(b)=1 / 2 \mathrm{~N}_{z} \mathrm{C}_{y} \mathrm{I}_{1 z} \mathrm{I}_{2 z}\left\{\cos \left(2 \pi \nu_{\mathrm{ZQC}} t_{1}\right) \pm \cos \left(2 \pi \nu_{2 \mathrm{QC}} t_{1}\right)\right\}
$$

where other multiple quantum terms (sine modulated) are removed by the phase alternation $\phi_{7}$.

The expressions in Eqs. [3] and [7] should be compared. In the experiment just described, glycine $\mathrm{H}_{\alpha}$ resonances are measured as the sum and the difference of their actual frequencies. The resulting twofold sensitivity gain is the direct effect of the complete coherence transfer that can be obtained between carbon magnetization and multiple quantum coherence in this case. Other effects, though, contribute to further enhance sen- sitivity and improve resolution at the same time, by narrowing the linewidth in $t_{1}$. In fact, on the one hand multiple quantum coherences excited in $t_{1}$ are unaffected by the geminal $J_{12}$ coupling, which therefore does not contribute to the linewidth and does not limit the duration of $t_{1}$. On the other hand, the relaxation of the multiple quantum coherence is more favorable since the direct dipolar interaction between the two protons, which is the dominant relaxation agent for single quantum proton terms, is not effective (8).

Due to the symmetry of the magnetization transfer, both proton operator terms in Eq. [7] are longitudinal and they carry a pure cosine modulation. The corresponding sine modulation necessary for quadrature detection cannot be conveniently accomplished in our scheme. Therefore, a real Fourier transformation is to be applied in $t_{1}$. To avoid the overlap of real peaks 
with peak images, the proton carrier can be set on one side with respect to all glycine ${ }^{1} \mathrm{H}_{\alpha}$ frequencies. As a result, ZQC and 2QC signals resonate in different regions of the spectrum, thereby allowing their simultaneous detection. If, on the other hand, the proton carrier is set in a central position, 2QC real peaks need to be separated from the corresponding quadrature images according to the TPPI method (8) and also edited with respect to the ZQC peaks, by storing separately the two data sets obtained by using $\phi_{7}=x,-x$ and $\phi_{7}=y,-y$. Then, adding and subtracting the two data sets provide separate subspectra containing ZQC and $2 \mathrm{QC}$ signals. Clearly ZQC real peaks still cannot be separated from corresponding images with this method, since zero-order coherences are not affected by the TPPI phase incrementation. However, the two proton frequencies can unambiguously be determined from the sum and the magnitude of the difference. Moreover, if a peak derived from ZQC is sufficiently close to the zero frequency limit of the spectrum, a certain degree of overlap with its quadrature image is bound to occur, depending on the linewidth. This phenomenon affects the lineshape and not the peak-integrated intensity.

In the setup that results in opposite signs for ZQC and 2QC peaks (see Eqs. [6] and [7]), the opposite initial phase necessarily makes the value of the observable net magnetization corresponding to the first theoretical $t_{1}$ point $\left(t_{1}=0\right)$ equal to zero. As is well known, the finite durations of real pulses, like, in particular $\phi_{6}$, which needs to invert alpha carbons without affecting carbonyls, do not in general allow the accurate acquisition of this first point. In this case, though, we can conveniently begin data acquisition at the second theoretical $t_{1}$ point by setting the initial delay equal to the dwell time in $t_{1}$, whereas the FID corresponding to $t_{1}=0$ can be produced during data processing by zeroing the first trace of the $2 \mathrm{D}$ data matrix. This trick eliminates the need for any zero- or firstorder phase corrections in the $t_{1}$ dimension, thereby producing spectra with reduced baseline distortions and $t_{1}$ ridges (9).

In the pulse sequence illustrated in Fig. 1 no water presaturation is used. Gradients are used to eliminate residual transverse water magnetization, whereas the last $90^{\circ}$ proton pulse is used to restore, at least partially, water magnetization along the $+z$-axis. This condition prevents cross saturation of amide protons. In general the use of water selective flipback pulses at appropriate times of pulse sequences guarantees optimum control on the solvent magnetization (10). Water flipback pulses can be introduced also in our pulse sequence, provided they are used outside the interval $\epsilon-$ pulse $\left(\phi_{5}\right)-t_{1}-$ pulse $\left(\phi_{7}\right)-$ $\epsilon$, where alpha protons are directly involved. A $90^{\circ}$ water selective pulse could be applied just before the carbon pulse $\left(\phi_{4}\right)$ with a phase opposite to $\phi_{5}$ in order to have water magnetization restored along the $+z$-axis before the $t_{1}$ period. Analogously, during the subsequent $\tau$ delay, a selective pulse with phase $\phi_{7}$ could set water magnetization along the $-z$-axis just before the next inversion pulse. The implementation of

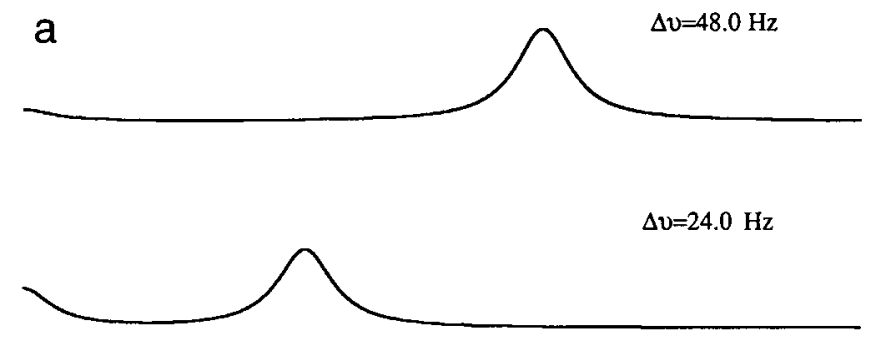

$\Delta v=16.8 \mathrm{~Hz}$
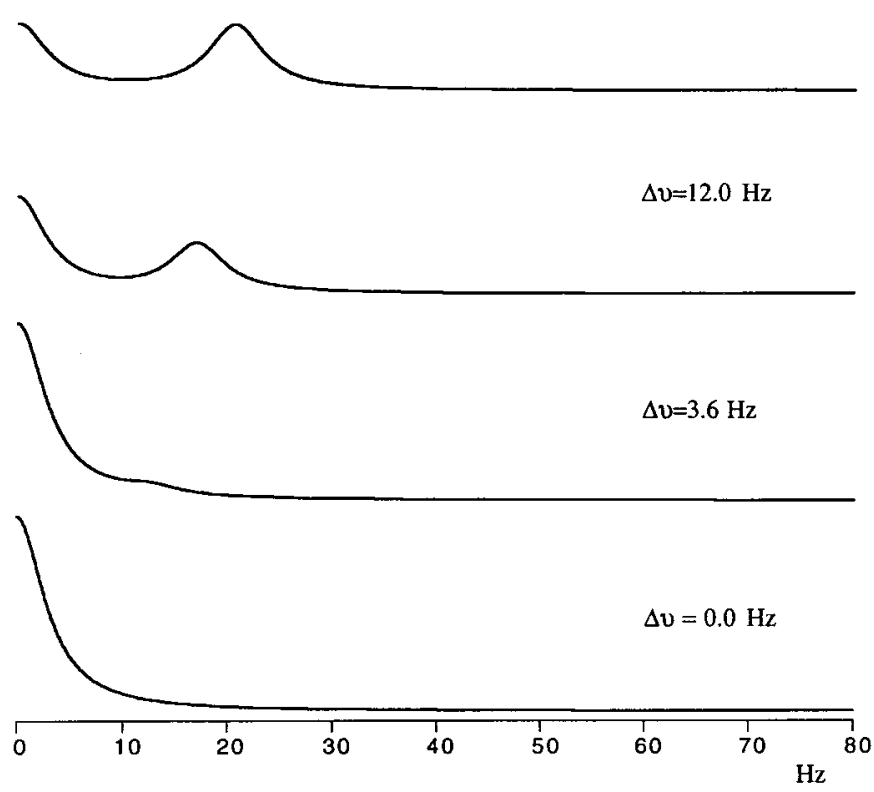

FIG. 3. (a) Simulated subspectra showing the relation between the ZQC and the corresponding zero-frequency peaks as a function of the strong coupling parameter $\theta=\frac{1}{2} \operatorname{arctg}\left\{J_{12} /\left(\nu_{1}-\nu_{2}\right)\right\}$. In the simulation a coupling constant $J_{12}=16 \mathrm{~Hz}$ is assumed throughout and the value of the chemical shift difference $\Delta \nu=\nu_{1}-\nu_{2}(\mathrm{~Hz})$ is varied. (b) Experimental subspectra corresponding to Gly 75 (bottom) and Gly 76 (top) NH frequencies. DQC peaks appear on the right. ZQC peaks appear on the left. Zero-frequency peaks appear truncated but with the correct area in both simulated and experimental spectra (see text).

water flipback pulses in the rest of the sequence is straightforward. The introduction of such pulses in the simple scheme illustrated in Fig. 1 would more efficiently restore the water magnetization to equilibrium $(+z$-axis) before data acquisition, thereby further optimizing sensitivity, particularly for glycines whose amide protons are more subject to cross saturation.

The high selectivity allows the execution of the experiment in a two-dimensional fashion, since the frequency dispersion of amide protons or nitrogens, separately, can be sufficient to resolve the spin systems, particularly if one considers that a much more adequate frequency resolution in the indirect dimension is obtained in a $2 \mathrm{D}$ experiment, compared to $3 \mathrm{D}$. 

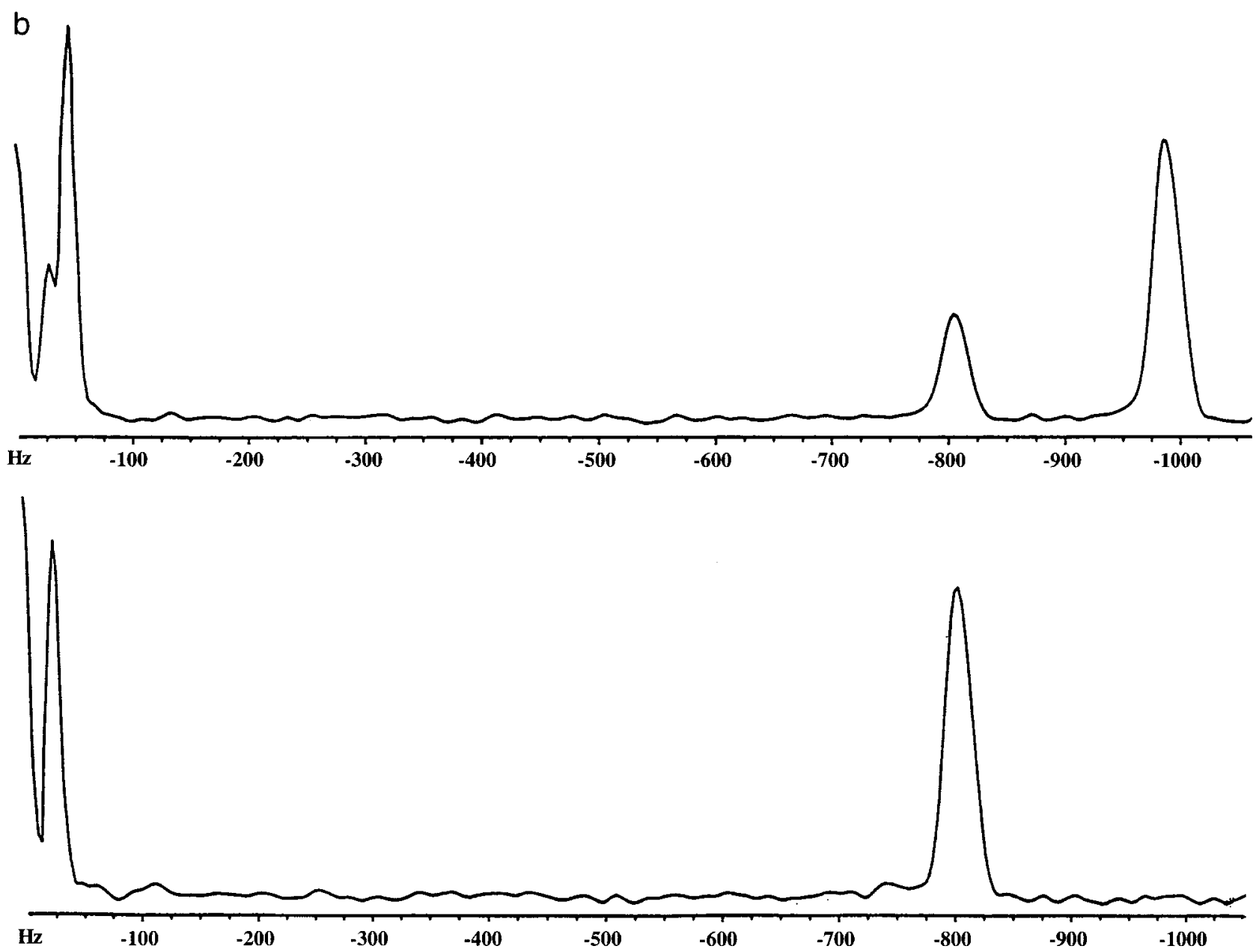

FIG. 3-Continued

\section{EXPERIMENTAL}

In Fig. 2, a two-dimensional version of the experiment is illustrated for a $2 \mathrm{mM}$ sample of human ubiquitin in water at $\mathrm{pH} 4.5$ and room temperature. In the spectrum, reported amide protons are correlated with alpha protons via their double- and zero-quantum frequencies, by incrementing $t_{1}$. All expected correlation peaks are present: ${ }^{1} \mathrm{H}_{\alpha}$ resonances of the six glycines are correlated with intraresidue $(i)$ and interresidue $(i+$ 1) amide proton resonances, via the sum and difference of their chemical shift, as measured with respect to the proton carrier. Since all ${ }^{1} \mathrm{H}_{\alpha}$ frequencies have the same sign, due to the carrier position (4.75 ppm), double quantum peaks appear in the upper region of the spectrum and zero quantum peaks in the lower region. The closer the chemical shifts for the two protons, the closer to zero the ZQC peak shows up in the spectrum. Therefore, magnetic equivalent (or nearly so) alpha-proton groups are promptly recognized. This constitutes a specific feature of the experiment that turns out to be quite useful for assignment purposes. In fact, the equivalence or simply the accidental coincidence (or nearly so) of alpha-proton chemical shifts is a source of ambiguity in experiments in which shifts are directly monitored, since the practical observation of a single correlation peak raises the question of whether it represents both alpha protons or just one of the two with the second correlation being missing or not easily distinguishable for whatever reason. Clearly the direct monitoring of the chemical shift difference (ZQC) unambiguously sets the issue. The peaks appear (advantageously) as singlets in this spectrum since homonuclear geminal coupling is not effective. The resulting chemical shifts are in general agreement with previously published data $(11,12)$.

In addition to glycines, only a very similar spin system $\mathrm{NH}-\mathrm{CH}_{2}-$ of arginine side chains also appears in the spectrum, on the right-hand side. The latter is easily distinguishable by the characteristic resonance position and multiplet pattern, due to the coupling interactions with the adjacent methilene group. 
Zero-quantum coherences exhibit somewhat narrower lines compared to double quantum coherences, as expected (8).

For Gly 75 we observe two peaks in the zero quantum region. This is clearly also visible in the correlation of the same ZQC with Gly $76 \mathrm{NH}$. The elucidation of this experimental detail calls for a brief digression.

\section{STRONG COUPLING EFFECTS}

Some particular consequences on the resulting spectrum are to be expected in the situation of strong coupling between the two geminal alpha protons. In this case, the expression in Eq. [5] does not contain only zero- and double-quantum coherences. The reason resides in the mixing of the spin states corresponding to zero total spin longitudinal component, operated by the Hamiltonian of the system

$$
\begin{aligned}
& \psi_{1}=\alpha \alpha ; \quad \psi_{2}=\cos \theta(\alpha \beta)+\sin \theta(\beta \alpha) ; \\
& \psi_{3}=-\sin \theta(\alpha \beta)+\cos \theta(\beta \alpha) ; \quad \psi_{4}=\beta \beta,
\end{aligned}
$$

where $\theta=\frac{1}{2} \operatorname{arctg}\left\{J_{12} /\left(\nu_{1}-\nu_{2}\right)\right\}$ represents the strong coupling parameter. In general the operators in Eq. [5] can be expanded in the eigenbase $(8)$

$$
\begin{aligned}
{\left[\mathrm{I}_{1}^{+} \mathrm{I}_{2}^{-}+\mathrm{I}_{1}^{-} \mathrm{I}_{2}^{+}\right]=} & {\left[\mathrm{I}^{+(2,3)}+\mathrm{I}^{-(2,3)}\right]\left[(\cos \theta)^{2}-(\sin \theta)^{2}\right] } \\
& +\left[\mathrm{I}^{(2,2)}-\mathrm{I}^{(3,3)}\right] \sin (2 \theta) \\
{\left[\mathrm{I}_{1}^{+} \mathrm{I}_{2}^{+}+\mathrm{I}_{1}^{-} \mathrm{I}_{2}^{-}\right]=} & {\left[\mathrm{I}^{+(1,4)}+\mathrm{I}^{-(1,4)}\right], }
\end{aligned}
$$

with $\mathrm{I}^{ \pm(2,3)}, \mathrm{I}^{ \pm(1,4)}$ representing ZQ and 2Q transitions, respectively, and with $\mathrm{I}^{(2,2)}, \mathrm{I}^{(3,3)}$ representing polarization operators (8).

Clearly the strong coupling does not have any effect on the $2 \mathrm{QC}$. We then consider only the evolution in $t_{1}$ of terms related to the ZQC:

$$
\begin{aligned}
\mathrm{I}^{ \pm(2,3)}\left(t_{1}\right) & =\mathrm{I}^{ \pm(2,3)} \exp \left\{\mp i 2 \pi \nu_{\mathrm{ZQC}} t_{1}\right\} ; \\
\mathrm{I}^{(2,2)}\left(t_{1}\right) & =\mathrm{I}^{(2,2)} ; \quad \mathrm{I}^{(3,3)}\left(t_{1}\right)=\mathrm{I}^{(3,3)},
\end{aligned}
$$

with

$$
\nu_{\mathrm{ZQC}}=\left[J_{12}^{2}+\left(\nu_{1}-\nu_{2}\right)^{2}\right]^{1 / 2} .
$$

By transforming back in the product base we get

$$
\begin{aligned}
\rho_{\mathrm{H}}\left(t_{1}\right)= & 1 / 2\left[\mathrm{I}_{1 y} \mathrm{I}_{2 y}+\mathrm{I}_{1 x} \mathrm{I}_{2 x}\right] \cos \left(2 \pi \nu_{\mathrm{ZQC}} t_{1}\right) \\
& \times\left\{\left[(\cos \theta)^{2}+(\sin \theta)^{2}\right]^{2}\right. \\
& \left.+[\sin (2 \theta)]^{2}\right\}+1 / 2\left[\mathrm{I}_{1 y} \mathrm{I}_{2 y}-\mathrm{I}_{1 x} \mathrm{I}_{2 x}\right] \\
& \times \cos \left(2 \pi \nu_{2 \mathrm{QC}} t_{1}\right)+\text { other terms. }
\end{aligned}
$$

TABLE 1

G lycine A Ipha-Proton Chemical Shifts as D etermined by Simultaneous Monitoring of Double- and Zero-Quantum Coherence Evolution

\begin{tabular}{lcccc}
\hline & $\nu_{2 \mathrm{QC}}(\mathrm{Hz})$ & $\nu_{\mathrm{ZQC}}(\mathrm{Hz})$ & $\delta_{1}(\mathrm{ppm})$ & $\delta_{2}(\mathrm{ppm})$ \\
\hline $\mathrm{G} 10$ & 800 & 370 & 4.32 & 3.58 \\
$\mathrm{G} 35$ & 745 & 106 & 4.11 & 3.90 \\
$\mathrm{G} 47$ & 1015 & 315 & 4.05 & 3.42 \\
$\mathrm{G} 53$ & 750 & 82 & 4.08 & 3.92 \\
$\mathrm{G}^{2} 5^{a}$ & 805 & 22 & 3.96 & 3.93 \\
$\mathrm{G}^{a}$ & 985 & 48 & 3.81 & 3.72 \\
\hline
\end{tabular}

Note. $\nu_{2 \mathrm{QC}}(\mathrm{Hz})=500.13\left\{\left[4.75-\delta_{1}(\mathrm{ppm})\right]+\left[4.75-\delta_{2}(\mathrm{ppm})\right]\right\} ; \nu_{1}(\mathrm{~Hz})$ $=500.13 \cdot \delta_{1}(\mathrm{ppm}) ; \nu_{2}(\mathrm{~Hz})=500.13 \cdot \delta_{2}(\mathrm{ppm}) ; \nu_{\mathrm{ZQC}}(\mathrm{Hz})=\left[\left(\nu_{1}-\nu_{2}\right)^{2}\right.$ $\left.+\mathrm{J}_{12}^{2}\right]^{1 / 2} ; J_{12}=15.8 \mathrm{~Hz}(\mathrm{G} 75) ; J_{12}=15.9 \mathrm{~Hz}(\mathrm{G} 76) ; 500.13=$ spectr. freq. $(\mathrm{MHz}) ; 4.75=\delta_{\text {carrier }}(\mathrm{ppm})$.

${ }^{a}$ See text.

Finally the proton pulse $\phi_{7}$ generates

$$
\begin{gathered}
\rho(b)=1 / 2 \mathrm{~N}_{z} \mathrm{C}_{y} \mathrm{I}_{1 z} \mathrm{I}_{2 z} \cdot\left\{\cos \left(2 \pi \nu_{\mathrm{ZQC}} t_{1}\right)\left[(\cos \theta)^{2}-(\sin \theta)^{2}\right]^{2}\right. \\
\left.+[\sin (2 \theta)]^{2} \pm \cos \left(2 \pi \nu_{2 \mathrm{QC}} t_{1}\right)\right\} .
\end{gathered}
$$

In practice $2 \mathrm{Q}$ transitions are not affected by the strong coupling, and therefore the position of the 2QC peaks always represents the sum of the two proton frequencies with unaltered intensity. On the other hand, the position of the $\mathrm{ZQC}$ peaks is given by the expression $\left[J_{12}^{2}+\left(\nu_{1}-\nu_{2}\right)^{2}\right]^{1 / 2}$ with intensity factor $\left[(\cos \theta)^{2}-(\sin \theta)^{2}\right]^{2}$. An additional peak at zero frequency is present with intensity factor [ $\sin (2$ $\theta)]^{2}$. From the ratio of the peak integrated intensities the parameter $\theta$ can be determined, and then from the ZQC peak position the accurate chemical shift difference between the two protons can be derived. Incidentally also the geminal coupling constant is evaluated. In the limit of weak coupling $\left(\theta \rightarrow 0^{\circ}\right)$ of course we regain the previous expressions and the zero frequency peak is absent. In the limit of infinite strong coupling $\left(\theta \rightarrow 45^{\circ}\right)$ only the zero-frequency peak is present with an intensity factor equal to unity. The relation between the ZQC and the zero frequency peak can be simulated as a function of the strong coupling parameter $\theta$. The result is illustrated in Fig. 3a. The zero-frequency peak appears truncated, since its maximum coincides with the limit of the spectrum. However, its area corresponds to the entire peak due to the symmetrical contribution of the peak quadrature image.

A conclusive and detailed interpretation of the experimental data illustrated in Fig. 2 can now be given. The relevant cross sections are reported in Fig. 3b. From the first cross section Gly 75 parameters can be derived and then, by subtracting the relative contribution of Gly 75 to the zero-frequency peak in the second, Gly 76 parameters can also be determined. Results 
are reported in Table 1, where data for all glycines are collected. Clearly if alpha protons are weakly coupled, then chemical shift calculation is trivial. For arginine side chains, frequency values are to be taken in the center of corresponding multiplets.

\section{CONCLUSIONS}

In summary, we have shown that a simple experiment can correlate amide proton and nitrogen resonances with ${ }^{1} \mathrm{H}_{\alpha}$ resonances specifically for glycine residues in a fully doubly labeled $\left({ }^{15} \mathrm{~N},{ }^{13} \mathrm{C}\right)$ protein. A unique feature of the experiment is the direct monitoring of the chemical shift difference (ZQC) of the alpha protons, which is quite useful to get unambiguous assignment in case of chemical shift degeneracy. Additionally in this experiment guanidine $\mathrm{NH}$ groups are correlated to delta protons of arginine side chains. Although a number of general experiments are available for assignment purposes of amino acids in doubly labeled proteins, this pulse sequence dedicated to glycines deserves some consideration, in our opinion, for its superior sensitivity and exquisite specificity.

\section{REFERENCES}

1. W. Boucher, E. D. Laue, S. Campbell-Burk, and P. J. Domaille, J. Am. Chem. Soc. 114, 2262 (91992).

2. L. E. Kay, M. Wittekind, M. A. McCoy, M. Friedrichs, and L. Mueller, J. Magn. Reson. 98, 443 (1992).

3. E. T. Olejniczak, R. X. Xu, A. M. Petros, and S. W. Fesik, J. Magn. Reson. 100, 444 (1992).

4. M. Rance, W. J . Chazin, C. Dalvit, and P. E. Wright, in "Methods in Enzymology" (N. Oppenheimer and T. L. J ames, Eds.), Vol. 176, p. 114, Academic Press, San Diego (1989).

5. M. Wittekind, W. J . Metzler, and L. Mueller, J . Magn. Reson. B 101, 214 (1993).

6. R. T. Cubb, V. Thanabal, and G. Wagner, J. Biomol. NMR 2, 203 (1992).

7. O. W. Sorensen, G. Eich, M. H. Levitt, G. Bodenhausen, and R. R. Ernst, Prog. NMR Spectrosc. 16, 163 (1983).

8. R. R. Ernst, G. Bodenhausen, and A. Wokaun, "Principles of Nuclear Magnetic Resonance in One and Two Dimensions," Clarendon Press, Oxford, 1987.

9. F. Ni, J. Magn. Reson. 99, 391 (1992).

10. S. Grzesiek and A. Bax, J. Am. Chem. Soc. 115, 12593 (1993).

11. D. L. Distefano and A. J. Wand, Biochemistry 26, 7272 (1987).

12. P. L. Weber, S. C. Brown, and L. Mueller, Biochemistry 26, 7282 (1987). 